
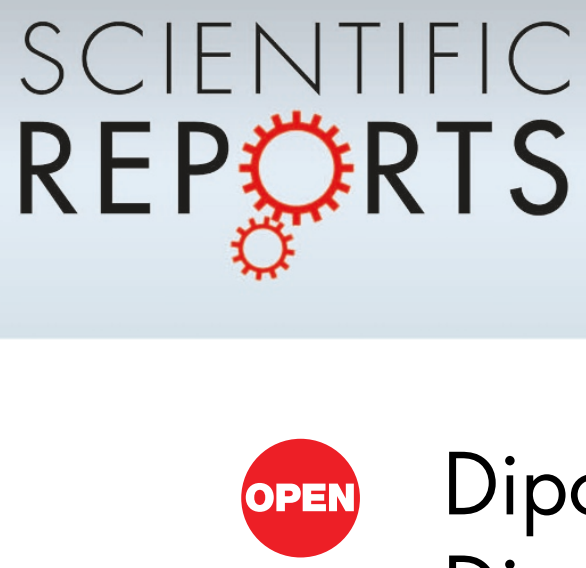

\title{
Dipolar Magnetism in Ordered and Disordered Low-Dimensional Nanoparticle Assemblies
}

SUBJECT AREAS:

MAGNETIC PROPERTIES AND MATERIALS

NANOPARTICLES

TWO-DIMENSIONAL MATERIALS

MAGNETIC DEVICES

Received

9 October 2012

\author{
M. Varón ${ }^{1,2 *}$, M. Beleggia ${ }^{3 *}$, T. Kasama ${ }^{3}$, R. J. Harrison ${ }^{4}$, R. E. Dunin-Borkowski ${ }^{3,5}$, V. F. Puntes ${ }^{2}$ \\ \& C. Frandsen'
}

'Department of Physics, Technical University of Denmark, 2800 Kgs. Lyngby, Denmark, ${ }^{2}$ Institut Català de Nanotecnologia, Campus U A B, 08193 Barcelona, Spain, ${ }^{3}$ Center for Electron Nanoscopy, Technical University of Denmark, 2800 Kgs. Lyngby, Denmark, ${ }^{4}$ Department of Earth Sciences, University of Cambridge, CB2 3EQ, Cambridge, United Kingdom, ${ }^{5}$ Ernst Ruska-Centre for Microscopy and Spectroscopy with Electrons and Peter Grünberg Institute, Research Centre Jülich, 52425 Jülich, Germany.

Accepted

18 January 2013

Published

6 February 2013

Correspondence and requests for materials should be addressed to C.F. (fraca@fysik.dtu.

Magnetostatic (dipolar) interactions between nanoparticles promise to open new ways to design nanocrystalline magnetic materials and devices if the collective magnetic properties can be controlled at the nanoparticle level. Magnetic dipolar interactions are sufficiently strong to sustain magnetic order at ambient temperature in assemblies of closely-spaced nanoparticles with magnetic moments of $\geq 100 \mu_{\mathrm{B}}$. Here we use electron holography with sub-particle resolution to reveal the correlation between particle arrangement and magnetic order in self-assembled 1D and quasi-2D arrangements of $15 \mathrm{~nm}$ cobalt nanoparticles. In the initial states, we observe dipolar ferromagnetism, antiferromagnetism and local flux closure, depending on the particle arrangement. Surprisingly, after magnetic saturation, measurements and numerical simulations show that overall ferromagnetic order exists in the present nanoparticle assemblies even when their arrangement is completely disordered. Such direct quantification of the correlation between topological and magnetic order is essential for the technological exploitation of magnetic quasi-2D nanoparticle assemblies.

\footnotetext{
* These authors contributed equally to this work.
}

$\mathrm{T}$ he assembly of magnetic nanoparticles into larger structures is of intense interest as an approach to engineer new materials and devices, such as strong magnets for high-end electronics ${ }^{1}$, logic gates ${ }^{2}$, thermally stable magnetic memories ${ }^{3}$ and soft magnetic materials for magnetic sensors ${ }^{4,5}$. It also provides new opportunities for shaping and diversifying components in devices, by making use of magnetic interactions between particles to build elaborate anisotropic structures such as chains and dendrites ${ }^{6-8}$. However, in order to reveal the full potential of these materials, quantification of the interplay between nanoparticle arrangements and magnetic order is essential.

Dipolar ferromagnetism ("magnetostatic alignment of all magnetic moments") was first predicted by Luttinger and Tisza for a face-centered cubic lattice of point dipoles". It was subsequently explored both theoretically and experimentally for nanoparticle assemblies, including particle chains ${ }^{10}$, ordered $2 \mathrm{D}$ arrays ${ }^{11-15}$, and $3 \mathrm{D}$ lattices ${ }^{16}$. Dipolar glasses in random 3D arrangements ${ }^{13,17-19}$ and dipolar antiferromagnetism in $2 \mathrm{D}$ arrays were also investigated ${ }^{20}$. However, local quantitative observations of dipolar magnetism in nanomaterials are lacking due to stringent experimental requirements. Consequently, the physical picture behind dipolar magnetism in nanoparticulate materials has developed with limited experimental support.

In conventional materials, dipolar magnetism is generally considered negligible, as the classical magnetostatic interactions between neighboring atoms with magnetic moments of only a few $\mu_{\mathrm{B}}$, a few $\AA$ apart, are too weak to explain magnetic ordering. Without electronic exchange coupling between atoms, solid-state magnetism ceases at non-vanishing temperatures. However, in "meta-materials" where atoms are replaced by nanoparticles, the "atomic" description of magnetism (i.e., strong exchange coupling and negligible dipolar interaction) breaks down as dipolar interactions may overwhelm inter-particle exchange coupling leading to dipolar magnetic ordering at $100-1000 \mathrm{~K}^{4,10-23}$.

An assembly of nanoparticles can be considered as a meta-material whose structure can range from crystalline when the underlying nanoparticle lattice is regular to amorphous when the particles are distributed randomly. The energy scale of dipolar interactions between magnetic nanoparticles varies with $\mu_{0} \mu^{2} / 4 \pi \mathrm{r}^{3}$ and is $\sim 2500 \mathrm{~K}$ for two $15 \mathrm{~nm}$ diameter Co particles with moments $\mu$ of $\sim 2 \cdot 10^{5} \mu_{\mathrm{B}}$ and a center-to-center separation of $\mathrm{r}=20 \mathrm{~nm}$. 
This value is comparable to the exchange coupling energy of $1388 \mathrm{~K}$ for $\mathrm{Co}$, and about four orders of magnitude larger than the dipolar coupling between two atomic spins at typical interatomic distances in a crystal.

Just as magnetic order and properties depend on the arrangement of atoms in a conventional material, so are dipolar magnetic order and properties expected to emerge from the geometry of an assembly of interacting nanoparticles in a nanoparticulate material; however, in such materials local structure-property relationships have not previously been quantified experimentally in detail. We have investigated dipolar magnetism at high spatial resolution by preparing a set of low-dimensional structures of self-assembled $15 \mathrm{~nm}$ cobalt particles, and using transmission electron microscopy and electron holography as primary imaging techniques. As a result of the anisotropic nature of the particles' magnetic dipole fields, elongated structures such as chains may form ${ }^{6-8,24}$ and local magnetic ordering can depend sensitively on the geometric arrangements of neighbouring particles: For instance, ferro- and antiferromagnetic dipolar ordering are predicted to occur for triangular and square particle lattices, respectively ${ }^{20}$ (see Fig. 1). Electron holography ${ }^{25,26}$ provides quantitative access to nanoscale magnetic field distributions with a spatial resolution well below the particle size. It is currently the only technique that can resolve non-invasively dipolar magnetism at this high resolution and sensitivity.

Here, we present the first direct observations of the local magnetic dipolar ordering in low-dimensional nanoparticle structures, and we correlate this to the exact geometric arrangement of the particles and to the magnetic history of the assemblies. The results clearly show the
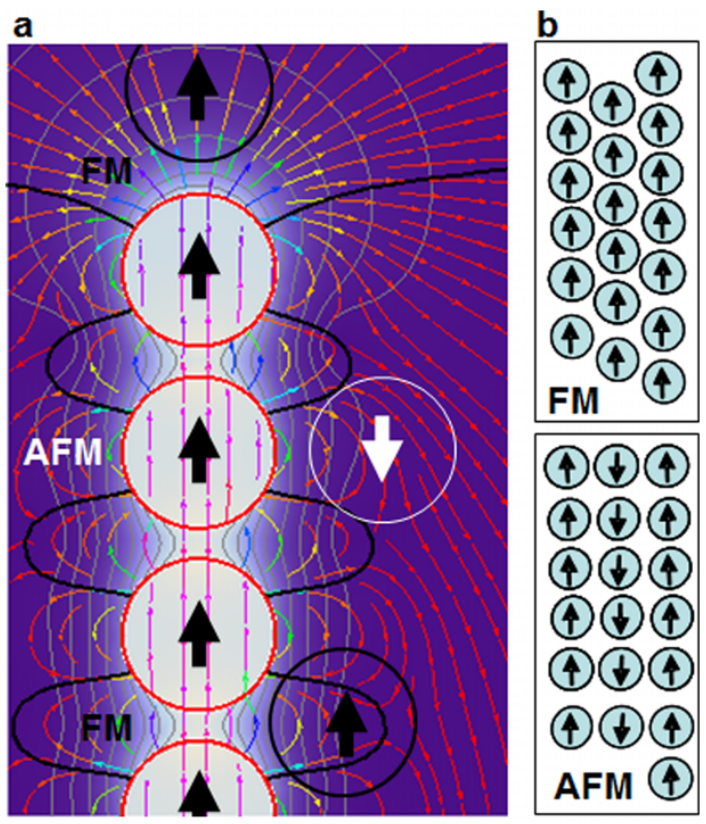

Figure 1 | Simulation and schematic diagrams illustrating magnetic dipolar fields and interactions between spherical nanoparticles.

(a) Magnetic field cross-section of the end of a chain of 10 uniformly magnetized spheres (red outline). The strongest field occurs at its ends. The fringing field gradient may guide additional particles to attach

preferentially at the ends of the chain in a ferromagnetic alignment (top particle with black outline). At the side of the chain the strong field between two particles favors ferromagnetic alignment if the added particle (black outline) is located within a relatively small region (labeled FM in a), while antiferromagnetic (AFM) alignment will occur otherwise (particle with white outline). For a freely moving particle the close-packed lattice with ferromagnetic alignment is energetically favored. (b) Schematic

illustrations of triangular (close-packed) and square lattices leading to FM and AFM dipole ordering, respectively. existence of dipolar magnetic ordering, which interestingly takes different forms depending on particle arrangement and magnetic history. Far from being "weak", dipolar magnetism in low-dimensional nanoparticle structures reveals a tendency to survive major degrees of structural disorder in the arrangement of the nanoparticles, to the point that we envision the existence of amorphous metamaterials with ferromagnetic character.

\section{Results}

We prepared assemblies of $15 \pm 2 \mathrm{~nm} \varepsilon$-Co particles with an oleic acid coating by evaporation of a colloid ${ }^{8,27}$ on a carbon substrate (with no external field applied). Figures 2 and 3 show transmission electron microscopy (TEM) bright-field images of particle assemblies. For chains wider than 1 particle across, particles are typically assembled into triangular (close-packed) lattices, although square lattice arrangements are also occasionally seen. The particles are separated by at least $2 \mathrm{~nm}$ (the size of the surfactant layer) providing full exchange decoupling.

We applied off-axis electron holography ${ }^{25,26}$ to map directly, and non-invasively, the projected magnetic field, $\boldsymbol{B}_{\text {in-plane, }}$ of the elongated nanoparticle assemblies with a nominal spatial resolution of $6.3 \mathrm{~nm}$ (fringe spacing is $2.1 \mathrm{~nm}$ ). Figure 2 shows color-coded holographic field maps acquired at remanence before and after applying an off-plane field of $\pm 2 \mathrm{~T}$, revealing the magnetic moment topography of the chains. In a single-particle-wide chain (i), simple ferromagnetic order exists already in the initial state (where chains may
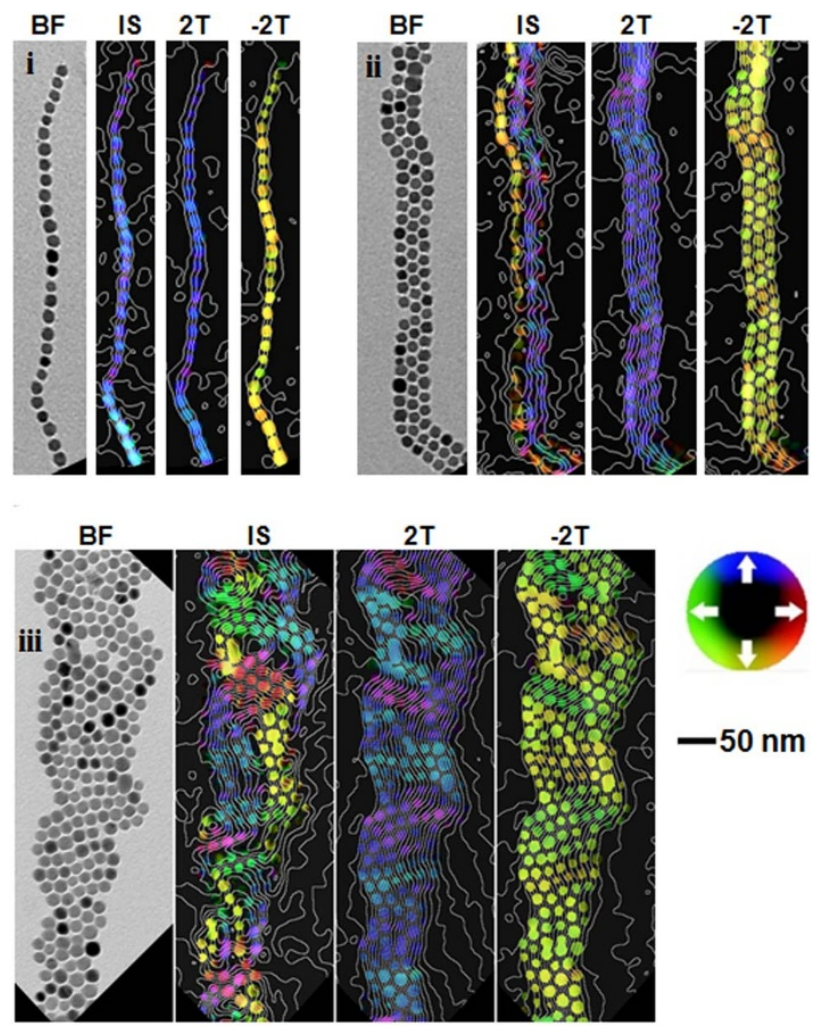

Figure $2 \mid$ Bright-field (BF) TEM images and holographic magnetic field maps of chains of $\sim 15 \mathrm{~nm}$ Co particles that are (i) one, (ii) three and (iii) multiple nanoparticles in width. Initial remanent magnetic states (IS) and magnetic states after application of a field of $\pm 2 \mathrm{~T}$ are shown. The white contours and the colors (described using the color wheel) provide a measure of the magnitude and direction of the projected magnetic field in the plane of the particles with a spatial resolution of $\sim 6 \mathrm{~nm}$. Each white contour represents a phase variation of $\pi / 64$, corresponding to $1 / 64$ of a flux quantum $\phi_{0}=2.07 \cdot 10^{-15} \mathrm{~T} \cdot \mathrm{m}^{2}$ enclosed between two adjacent contours. 
a

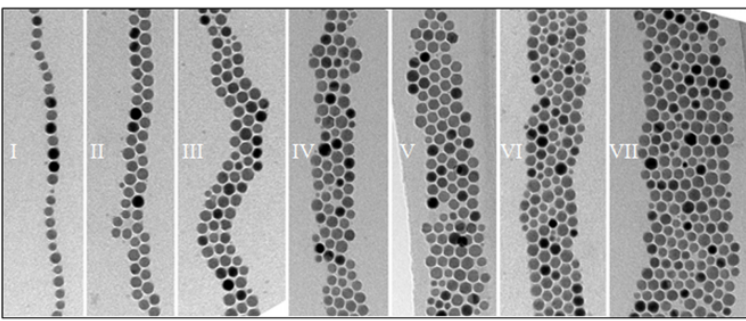

b

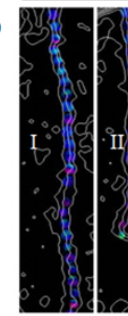

$\mathrm{CN}$

$\begin{array}{lll}\sigma_{g} & 0.14 & 0.15\end{array}$

$\begin{array}{lll}\sigma_{\mathrm{m}}(\mathrm{rad}) & 0.16 & 0.27\end{array}$

$\begin{array}{lll}M & 0.97 \quad 0.95\end{array}$
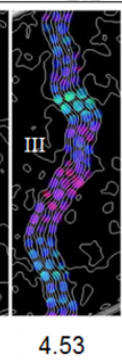

0.15

0.15
0.44

0.87

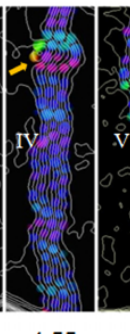

4.55

0.15

0.34

0.88
4.81

0.16

0.29

0.90

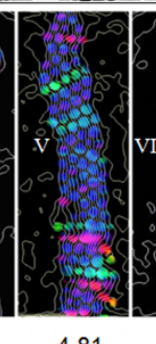

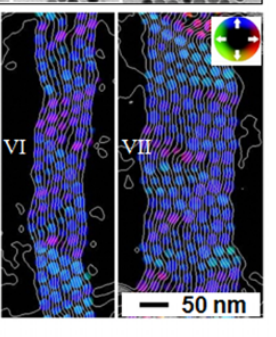

5.65

0.21

0.30

0.94 a

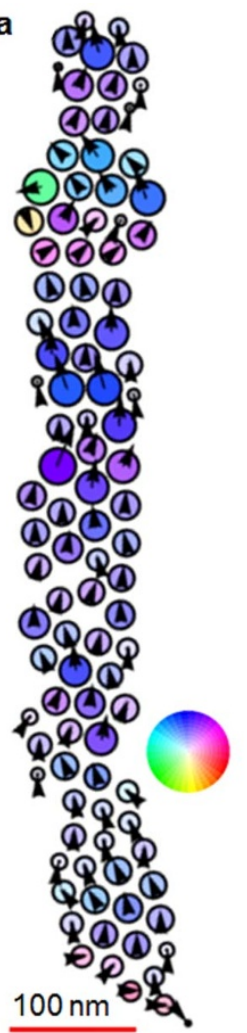

b $\quad \Delta \theta=\pi / 2$

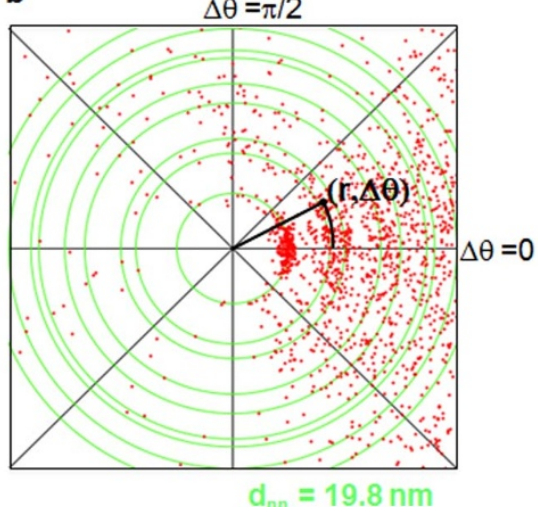

Figure 3 | (a) Bright-field TEM images and (b) holographic magnetic field maps obtained from $\sim 15 \mathrm{~nm}$ Co particle chains of increasing width numbered I-VII at remanence after magnetic saturation. $\mathrm{CN}=$ coordination number ( $=$ average number of nearest neighbors), $\sigma_{\mathrm{g}}=$ geometric disorder, $\sigma_{\mathrm{m}}=$ magnetic disorder (angle measured in radians), $\mathrm{M}=$ relative net magnetic moment of chain. Phase contour spacing as in Fig. 2.

have been exposed only to the weak stray field, $\sim 6 \mathrm{mT}$, of the microscope column while inserting the sample). Chains wider than one particle show more complicated initial states. In a 3-particle-wide chain (ii), ferromagnetic alignment is observed only for two of the three strands while antiferromagnetic alignment is found between these two strands and the third one. The antiferromagnetic alignment seems to be related to cases of square lattice arrangement between some of the particles. In a 6-particle-wide chain (iii), several flux-closed regions with domain widths of up to 5 particles are observed in the initial state. No out-of-plane cores, typical for regular vortices in continuous thin films, are found as a result of the absence of inter-particle exchange interactions. At remanence after magnetic saturation in a field of $\pm 2 \mathrm{~T}$, overall dipolar ferromagnetic order is observed along the direction of chains i-iii, with only local shortrange variations ("ripples") of the moment orientations in chains ii and iii.

In order to quantify dipolar ferromagnetic order in the nanoparticle structures we examine a set of chains (I-VII, Fig. 3) with widths of 1-8 particles after applying a field of $2 \mathrm{~T}$. The average number of nearest neighbour particles (coordination number, $\mathrm{CN}$ ) of each particle ranges from 2 for the single-particle chain (I) to 5.65 for the wider chains corresponding to quasi-2D particle arrangements $(\mathrm{CN}$ $=6$ for infinite close-packed 2D lattices).

From the projected field maps (Fig. 3b) we estimate the magnitude and orientation of the magnetic moment of each individual particle (Fig. 4a shows this for chain IV) and correlate it with the geometrical arrangement of the particles. For each pair of particles in each chain we measure the spatial separation $r$ between their centers and the angular difference $\Delta \theta$ between their moments. Figure $4 \mathrm{~b}$ shows the distribution $(r, \Delta \theta)$ for chain IV. We found for each chain the dimensionless geometric order parameter ratio $\sigma_{\mathrm{g}}=\sigma_{\mathrm{nn}} / \mathrm{d}_{\mathrm{nn}}$, where $\mathrm{d}_{\mathrm{nn}}$ is the average nearest neighbor distance and $\sigma_{n n}$ is the spread in $d_{n n}$. We also found the degree of local magnetic disorder $\sigma_{\mathrm{m}}$ from the spread in $\Delta \theta$ between nearest neighbors, and the overall relative net moment $\mathrm{M}$ per chain as the actual vector sum of all moments in a chain divided by the nominal sum of all moments.

Figure $4 \mid$ Results obtained from measurement of chain IV in Fig. 3. (a) Estimated magnetic moment distribution, (b) radial-angular $(r, \Delta \theta)$ distribution function, where $r$ is distance between two magnetic moments (particle centers) and $\Delta \theta$ is the angular difference in their moment orientations, measured for all pairs of moments in the chain. The thin green circular lines superimposed on (b) indicate the expected locations of the first few neighbouring peaks for a $2 \mathrm{D}$ close-packed lattice with a measured nearest neighbour distance $\mathrm{d}_{\mathrm{nn}}$ of $19.8 \mathrm{~nm}$.

As given in Fig. 3, we find that $\mathrm{M}$ indicates almost perfect ferromagnetic order $(M=0.97)$ for the single particle chain; also, $\sigma_{m}$ is small. For wider chains, $\mathrm{M}$ is $\sim 10 \%$ lower and $\sigma_{\mathrm{m}}$ is about two times larger, but $\sigma_{\mathrm{m}}$ and $M$ reveal that the magnetic order is strongly ferromagnetic on both short- and long-range scales. All chains have a similar geometric disorder $\sigma_{\mathrm{g}}$ of around $0.17 \pm 0.04$. The spread in particle size (relative to mean particle size) gives values similar to those of $\sigma_{\mathrm{g}}$ for all chains. This suggests that the geometric disorder may be driven primarily by the size distribution, and that the packing of the particles on the substrate is almost as dense as possible for the given particle size distribution.

In order to simulate magnetic order in the chains, we used a computational framework based on an adaptation of the LandauLifshitz-Gilbert equations that describe semi-classically the dynamics of interacting magnetic moments inside materials ${ }^{28}$. Starting from the measured coordinates and radii of the particles, and assuming an initial in-plane alignment that mimics the external field applied to saturate the structures, we calculated the expected ground states upon removal of the field for particles with zero magnetic anisotropy. Despite edge effects and a few minor discrepancies between simulations and observations, for example in the exact position of the vortex in chain IV, the simulations confirm magnetic ordering as being long-range dipolar ferromagnetic.

We also simulated relaxation from an initial random distribution of moments, obtaining results similar to the initial magnetic states, with shorter range magnetic order that includes flux-closed regions that are a few particles in width for the chains wider than 1 particle. In 
a

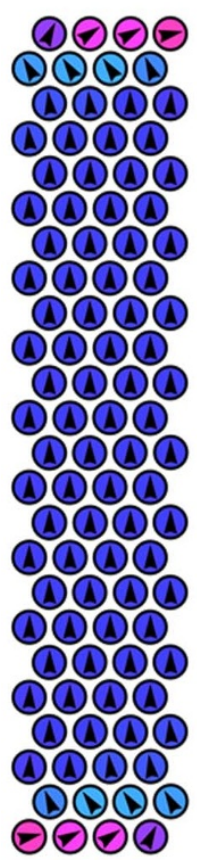

$\sigma_{\mathrm{g}}=0$ b

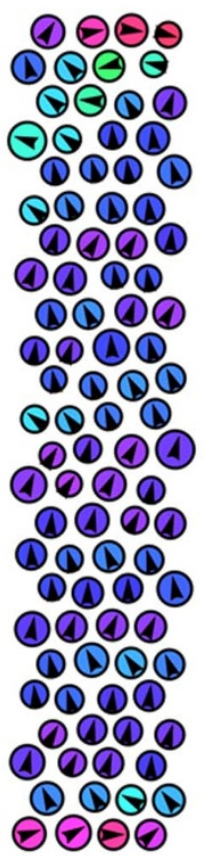

$\sigma_{\mathrm{g}}=0.2$ c

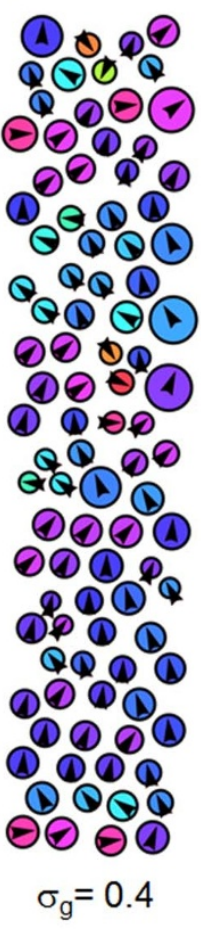

d

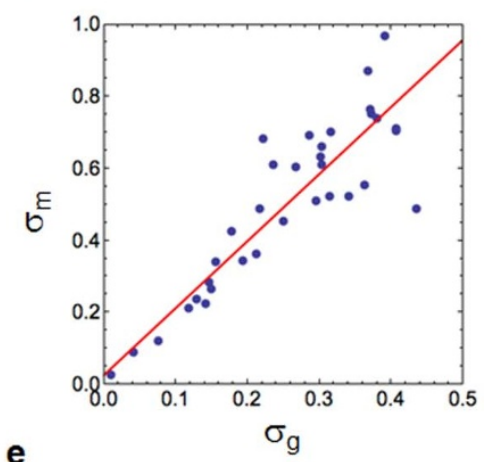

e

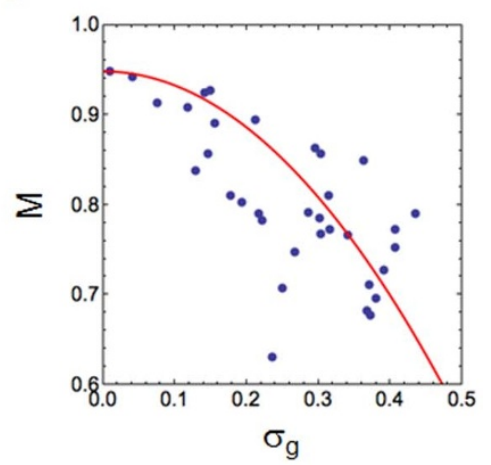

Figure $5 \mid$ Simulations of magnetic order in chains with increasing lattice disorder. (a) $4 \times 18$ particle chain with perfect triangular lattice $\left(\sigma_{\mathrm{g}}=0\right)$, (b, c) $4 \times 18$ particle chain with disordered particle arrangement $\left(\sigma_{\mathrm{g}}=0.2-0.4\right)$, (d) plot of the magnetic disorder $\sigma_{\mathrm{m}}$ vs. geometric disorder $\sigma_{\mathrm{g}}$, obtained from a set of 35 simulations with varying $\sigma_{\mathrm{g}}$, (e) plot of the normalized net magnetic moment $\mathrm{M}$ as a function of geometric disorder $\sigma_{\mathrm{g}}$. Color-code as shown in Fig. 4.

these cases, many degenerate magnetic states exist. This correspondence between simulated and observed states together with the presence of complex initial magnetic states suggest that the assembly into chains is not entirely magnetically driven.

The simulations were used also to assess structures that were not available experimentally by artificially increasing the degree of geometrical (lattice) disorder for a finite lattice of dipoles by adding a random component to the position and size of each nanoparticle. An example of a chain with dimensions of $4 \times 18$ nanoparticles $(\mathrm{CN}=$ $4.85)$, is shown in Figs. 5a-c, where the initially regular triangular lattice $\left(\sigma_{\mathrm{g}}=0\right)$ is distorted gradually up to $\sigma_{\mathrm{g}}=0.4$ such that the particle arrangement is indistinguishable from an amorphous structure, i.e. order at nearest neighbors is lost. Even in this extreme case, simulations of the magnetic saturation remanence show that $\mathrm{M}$ has decreased only from 0.95 to 0.70 . $\sigma_{\mathrm{m}}$ corresponds to an angular spread of about 45 degrees. Results of the simulations for intermediate values of $\sigma_{\mathrm{g}}$ are summarized in Figs. 5d,e, revealing a direct proportionality between geometrical and magnetic disorder $\left(\sigma_{g}\right.$, $\left.\sigma_{\mathrm{m}}\right)$ and a slow, close to parabolic, decay of $\mathrm{M}$ with geometric disorder $\sigma_{\mathrm{g}}$. The persistent ferromagnetism in the quasi-2D structures appears contrary to the spin-glass behavior often expected in disordered nanoparticle-systems.

\section{Discussion}

For the arrangements of $15 \mathrm{~nm}$ Co particles studied here, our results show that short-range magnetic order with small domains dominates in the initial states, with the local magnetic order (ferromagnetic vs. antiferromagnetic) depending on the particle lattice (triangular vs. square). In contrast, at remanence after saturation, overall dipolar ferromagnetic order is extremely persistent even in case of a nontriangular lattice. We interpret our results as supporting the existence of amorphous dipolar ferromagnets: i.e., dipolar ferromagnetism exists in $1 \mathrm{D}$ to quasi-2D structures even in the absence of underlying crystallinity. These observations are contrary to the magnetic glass behavior found in random 3D particle arrangements ${ }^{13,17-19}$ and are of direct relevance for the use of quasi-2D nanoparticle assemblies in magnetic devices such as thin elongated memories, tunnel junctions, and sensors.

\section{Methods}

Spherical $\varepsilon$-Co nanoparticles, $\sim 15 \mathrm{~nm}$ in diameter and coated with oleic acid, were synthesized using a method adapted from Ref. 27, which involves thermal decomposition of a cobalt precursor in a mixture of hot organic solvent and surfactants at high temperature and under argon atmosphere using a Shlenkline setup to avoid oxidation. Cobalt carbonyl $\left(\mathrm{Co}_{2}(\mathrm{CO})_{8}\right.$, moistened with $5-10 \%$ hexane), trioctylphosphine oxide (TOPO, 99\%), oleic acid (OA, 99\%) and 1,2-dichlorobenzene anhydrous (DCB, 99\%), were purchased from Sigma-Aldrich and used as received without further purification. All chemicals were stored and prepared for synthesis in a UNIlab MBraun glovebox. The cobalt precursor solution $\left(0.54 \mathrm{~g}^{\circ} \mathrm{Co}_{2}(\mathrm{CO})_{8}\right.$ dissolved in $3 \mathrm{ml}$ of DCB anhydrous) was injected rapidly into a refluxing DCB anhydrous solution $\left(181^{\circ} \mathrm{C}\right)$ containing the surfactant mixture $(15 \mathrm{ml}$ of DCB in the presence of $0.2 \mathrm{~g}$ of OA and $0.15 \mathrm{~g}$ of TOPO). The solution was refluxed at $181^{\circ} \mathrm{C}$ for 15 min under continuous stirring and then gradually cooled down before being transferred to an argon filled vial. One drop of the cobalt nanoparticle solution was then deposited onto a holey carbon-coated copper TEM grid and left to dry inside a glove box.

Off-axis electron holograms were acquired at $300 \mathrm{kV}$ in Lorentz mode in a Titan 80-300 ST TEM with a charge-coupled device (CCD) camera and an electron biprism operated typically at $200 \mathrm{~V}$. The experiments were performed at room temperature, and the acquisition time was 6-12 s. Initial magnetic states were recorded after loading the as-prepared samples into the TEM in almost field-free (below $6 \mathrm{mT}$ ) conditions. The direction of magnetization in each chain was subsequently reversed in-situ in the TEM by tilting the sample by $\pm 60^{\circ}$ and turning on the conventional microscope objective lens to apply a magnetic field of $2 \mathrm{~T}$ to the sample, parallel to the direction of the electron beam. The objective lens was then turned off and the sample tilted back to $0^{\circ}$ for hologram acquisition in field-free conditions and with the particles at remanence. Following this procedure, the different holograms were recorded with the chains magnetized in opposite directions. The mean inner potential was separated from the magnetic potential as described in [25].

The magnitude and orientation of each moment was measured by integrating the phase gradient components within each nanoparticle in each chain. For uniformly magnetized spherical particles, the integrated phase gradient lies in the same direction 
and is directly proportional to the magnetic moment, with a factor of proportionality equal to $\pi \mu_{0} /\left(2 \phi_{0}\right)=0.954 \mathrm{rad} \cdot \mathrm{A}^{-1} \cdot \mathrm{nm}^{-1}$, where $\mu_{0}$ is the vacuum permeability, and $\phi_{0}=2.07 \cdot 10^{-15} \mathrm{~T} \cdot \mathrm{m}^{2}$ is a flux quantum. The measurement is generally perturbed by the presence of neighboring particles proportionally to the magnitude and orientation of the field generated by surrounding particles. However, if the system is at equilibrium, no torque is acting on any of the moments so that the local field and each moment are aligned; as a consequence, we do not expect significant perturbations in the measured orientation.

The magnetic relaxation of the chains was simulated by an iterative approach based on a simplified version of the LLG equations, in which precession of moments around the local magnetic field was ignored (high damping coefficient). A simulation proceeds in steps: 1) the simulated structure is created by assigning coordinates and diameters of the nanoparticles from an experimental micrograph; 2) each particle is assumed to be spherical, and with a magnetic moment proportional to its volume and with no magnetic anisotropy; 3 ) the initial orientation is chosen as either "saturated " where all moments are aligned (with a small random component), or "random" where all moments are pointing randomly; 4) the local magnetic field acting on the i-th particle is calculated as superposition of the magnetic fields generated by the remaining N-1 particles; 5) the i-th moment is rotated towards the local field by an amount proportional to the torque, i.e. to the cross product between moment and local field; 6) a new moment topography is obtained, and the procedure is repeated until the total residual absolute torque becomes smaller than a pre-defined threshold.

1. Jones, N. The pull of stronger magnets. Nature 472, 22-23 (2011).

2. Cowburn, R. P. \& Welland, M. E. Room temperature magnetic quantum cellular automata. Science 287, 1466-1468 (2000).

3. Zeng, H., Li, J., Liu, J. P., Wang, Z. L. \& Sun, S. H. Exchange-coupled nanocomposite magnets by nanoparticle self-assembly. Nature 420, 395-398 (2002).

4. Bedanta, S. et al. Overcoming the dipolar disorder in dense CoFe nanoparticle ensembles: Superferromagnetism. Phys. Rev. Lett. 98, 176601 (2007).

5. Chemla, Y. R. et al. Ultrasensitive magnetic biosensor for homogeneous immunoassay. Proc. Natl Acad. Sci. USA 97, 14268-14272 (2000).

6. Butter, K., Bomans, P. H. H., Frederik, P. M., Vroege, G. J. \& Philipse, A. P. Direct observation of dipolar chains in iron ferrofluids by cryogenic electron microscopy. Nature Mater. 2, 88-91 (2003).

7. Ku, J. Y., Aruguete, D. M., Alivisatos, A. P. \& Geissler, P. L. Self-assembly of magnetic nanoparticles in evaporating solution. J. Am. Chem. Soc. 133, 838-848 (2011).

8. Varón, M. et al. Dipolar driven spontaneous self assembly of superparamagnetic Co nanoparticles into micrometric rice-grain like structures. Langmuir $\mathbf{2 6}$, 109-116 (2010).

9. Luttinger, J. M. \& Tisza, L. Theory of dipole interaction in crystals. Phys. Rev. 70, 954-964 (1946).

10. Sugawara, A. \& Scheinfein, M. R. Room-temperature dipole ferromagnetism in linear-self-assembling mesoscopic Fe particle arrays. Phys. Rev. B 56, R8499-R8502 (1997).

11. Russier, V. Calculated magnetic properties of two-dimensional arrays of nanoparticles at vanishing temperature. J. Appl. Phys. 89, 1287-1294 (2001).

12. Puntes, V. F., Gorostiza, P., Aruguete, D. M., Bastus, N. G. \& Alivisatos, A. P. Collective behaviour in two-dimensional cobalt nanoparticle assemblies observed by magnetic force microscopy. Nature Mater. 3, 263-268 (2004)

13. Lisiecki, I., Parker, D., Salzemann, C. \& Pileni, M. P. Face-centered cubic supra-crystals and disordered three-dimensional assemblies of $7.5 \mathrm{~nm}$ cobalt nanocrystals: Influence of the mesoscopic ordering on the magnetic properties. Chem. Mater. 19, 4030-4036 (2007).

14. Yamamoto, K., Hogg, C. R., Yamamuro, S., Hirayama, T. \& Majetich, S. A. Dipolar ferromagnetic phase transition in $\mathrm{Fe}_{3} \mathrm{O}_{4}$ nanoparticle arrays observed by Lorentz microscopy and electron holography. Appl. Phys. Lett. 98, 072509 (2011).
15. Yamamoto, K. et al. Direct visualization of dipolar ferromagnetic domain structures in Co nanoparticle monolayers by electron holography. Appl. Phys. Lett. 93, 082502 (2008).

16. Chen, J. et al. Collective dipolar interactions in self-assembled magnetic binary nanocrystal superlattice membranes. Nano Lett. 10, 5103-5108 (2010).

17. Mørup, S. \& Christiansen, G. Influence of magnetic anisotropy on the superferromagnetic ordering in nanocomposites. J. Appl. Phys. 73, 6955-6957 (1993).

18. Jonsson, T. et al. Aging in a magnetic particle system. Phys. Rev. Lett. $\mathbf{7 5}$, 4138-4141 (1995).

19. Djurberg, C. et al. Dynamics of an interacting particle system: Evidence of critical slowing down. Phys. Rev. Lett. 79, 5154-5157 (1997).

20. Politi, P. \& Pini, M. G. Dipolar interaction between two-dimensional magnetic particles. Phys. Rev. B 66, 214414 (2002).

21. Scheinfein, M. R., Schmidt, K. E., Heim, K. R. \& Hembree, G. G. Magnetic order in two-dimensional arrays of nanometer-sized superparamagnets. Phys. Rev. Lett. 76, 1541-1544 (1996)

22. Tomita, S. et al. Tuning magnetic interactions in ferromagnetic-metal nanoparticle systems. Phys. Rev. B 71, 180414 (2005).

23. Majetich, S. A. \& Sachan, M. Magnetostatic interactions in magnetic nanoparticle assemblies: energy, time and length scales. J. Phys. D. Appl. Phys. 39, R407-R422 (2006).

24. Salgueiriño-Maceira, V., Correa-Duarte, M. A., Hucht, A. \& Farle, M One-dimensional assemblies of silica-coated cobalt nanoparticles: Magnetic pearl necklaces. J. Magn. Magn. Mater. 303, 163-166 (2006).

25. Dunin-Borkowski, R. E. et al. Magnetic microstructure of magnetotactic bacteria by electron holography. Science 282, 1868-1870 (1998).

26. Dunin-Borkowski, R. E. et al. Off-axis electron holography of magnetic nanowires and chains, rings, and planar arrays of magnetic nanoparticles. Microsc. Res. Tech. 64, 390-402 (2004).

27. Puntes, V. F., Krishnan, K. M. \& Alivisatos, A. P. Colloidal nanocrystal shape and size control: The case of cobalt. Science 291, 2115-2117 (2001).

28. Gilbert, T. L. A phenomenological theory of damping in ferromagnetic materials. IEEE Trans. Magn. 40, 3443-3449 (2004).

\section{Acknowledgements}

M.V. thanks the Spanish MICINN for financial support through scholarship BES-2007-17164. CF thanks the Danish Research Council for Independent Research for funding (Steno stipend).

\section{Author contributions}

M.V., T.K., M.B., V.F.P., R.E.D.B. and C.F. conceived the project and designed the experiments. M.V. prepared the samples. T.K. and M.V. carried out the holography experiments and processed the images. R.J.H. determined particles sizes and positions. M.B. did the simulations and most of the data analysis and interpretation. All authors discussed the results. C.F. and M.B. wrote the main text of the manuscript, but all authors contributed to the writing.

\section{Additional information}

Competing financial interests: The authors declare no competing financial interests.

License: This work is licensed under a Creative Commons

Attribution-NonCommercial-NoDerivs 3.0 Unported License. To view a copy of this license, visit http://creativecommons.org/licenses/by-nc-nd/3.0/

How to cite this article: Varón, M. et al. Dipolar Magnetism in Ordered and Disordered Low-Dimensional Nanoparticle Assemblies. Sci. Rep. 3, 1234; DOI:10.1038/srep01234 (2013). 